\title{
Prioritization and transparency in software development: An action research in public administration
}

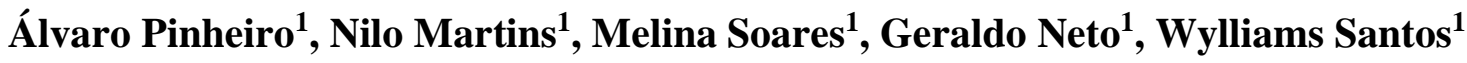 \\ ${ }^{1}$ Pernambuco Polytechnic School, University of Pernambuco, Recife, Brazil. \\ afpdecomp.poli.br, jnms@ecomp.poli.br, msdsdecomp.poli.br, \\ gtgn@ecomp.poli.br, wbseupe.br
}

\begin{abstract}
The prioritization of requirements is a critical activity in quality assurance. However, in public institutions, prioritization faces conflicts with financial constraints. Problem: Increasing the complexity of requirements. Objective: Proposition of a Kanban method to improve the transparency of activities and facilitate prioritization. Method: An action research carried out in two cycles at the Attorney General's Office of the State of Pernambuco, involving the development team and public managers. Result: The presentation of the method's effectiveness as a mechanism facilitating transparency and as a support to management in prioritization.
\end{abstract}

\section{Introduction}

According to Hujainah and Bakar (2018), requirements prioritization is one of the most important phases of software development, involving critical decision-making activities that determine product quality, in many cases being responsible for the success or failure of a project.

Public organizations focused on software development often have a high volume of requests for à new software requirements, often higher than their development capability. Thus, prioritizing these requirements becomes a crucial activity. Products with high business value are delivered to the public administration Hujainah and Bakar (2018).

According to Babar et al. (2011), the prioritization of requirements is a complex activity. In public agencies, specific factors contribute to this increase in complexity, and they are: High number of customers from different areas with conflicting priorities about their requirements, low visibility of high management on the actual production capacity of the development team, and on the requirements that are already being met.

This article presents the results of the action research that includes implementing the Kanban method through an online tool in a public agency of the State of Pernambuco to improve the transparency of the activities of the development team and facilitate the prioritization of requirements by senior management.

This research action was carried out in two cycles with the Attorney General's Office of the State of Pernambuco (PGE/PE) and involved both the technical team responsible for the development and maintenance of systems, as well as the high management responsible for prioritizing the requirements for this team. The first cycle 
focused on implementing the Kanban method in an online tool with the technical team and presentation to all stakeholders. The second cycle was focused on the validation and legitimation of the need to apply the transparency technique to the prioritization of requirements. The transitions between the different steps are discussed in detail, along with the identified obstacles, benefits, and lessons learned.

The rest of the article is organized as follows. In Section 1, the introduction is performed, in Section 2, presents Kanban's theoretical framework to be evaluated, prioritization and public administration. In Section 3, the cycles of the research-action are presented. In Section 4, the general results obtained during the application of the research-action are presented. Section 5 presents the conclusions.

\section{Theoretical Foundation}

This section addresses the Kanban method, prioritization, and public administration.

\subsection{Kanban}

Kanban is mentioned as a method that allows increased productivity Anderson (2010) and Petersen (2014) rapid response to changes in requirements Taibi (2017), which is ideally suited to public agencies, as the requirements are dynamic and the existence of lean teams. According to Anderson (2010), Kanban is described as a method of evolutionary change that uses a visualization system and other tools to catalyze the introduction of ideas, being an evolutionary and incremental process. Thus, Kanban adapts to the agile principles desired and necessary for development teams, being a tool to optimize results, adding value through the focus on flow management. To measure and manage the flow, some techniques are presented: the Kanban framework; the value stream map; the cumulative flow diagram; and the balance status chart line Petersen (2014).

The Kanban method is based on lean principles that try to prevent and remove waste from the production process. It was created as a lean tool for managing manufacturing processes and operations but was also applied in software development and is considered as an adaptable method that focuses on cost savings. If scope changes are required, Kanban works best because it focuses on minimizing and limiting work in progress.

\subsection{Prioritization}

Lehtola (2004) prioritization is intended to provide management capability to software products, determining which requirements are candidates to be included in each delivery. Another aspect is prioritization, which aims to minimize risks during development. The most important or high-risk requirements are implemented first, and there are several methods to assess prioritization.

In software development management, according to Lehtola (2004), there are subprocesses, such as portfolio management, in which product development is defined based on the external environment. Requirements management focuses on the software 
requirements that are candidates for a product that must be gathered and organized so that it allows management in the delivery planning activity. These requirements are prioritized and selected so that delivery can be prepared. Therefore, one of the main steps in release planning is prioritization.

A practical method, according to Karlsson (1997) and Berander (2005), to prioritize software requirements is the cost-value approach because this approach uses the basic idea of determining for each candidate requirement, what the cost of implementation is, and what its value is. The assessment of values and costs for requirements is based on the basic idea that, for all candidate requirement pairs, a person evaluates a value or cost by comparing a requirement of one pair with the other. In this way, stakeholders use the cost-value diagram as a conceptual map for analysis, and the coordinator or manager prioritizes the requirements.

According to the researchers read here, some of the existing prioritization techniques are Planning game (PG); 100-point method (100P); MoSCoW method (MM). As is the standard, today the agencies make use of agile methods because the dynamism of the requirements and the quantity of components prevent another form of management. Prioritization is based on the greater need to solve the problem, making partial use of the planning game technique to put priority on paper.

According to Beck (2000) and Berander (2005), the planning game comprises a meeting that takes place once per iteration, usually once a week. The planning process is divided into two parts: Posting Planning, which is focused on determining which requirements are included in which short-term postings and when they should be delivered, and iteration planning, which plans the activities of developers.

The 100-point method, described by the authors, consists of an orderly voting method that can have multiple winners who need to promote a proportional relationship of what is believed to be a priority by the team with what is specified as a priority by the client.

According to McIntyre (2016), the MoSCoW Method is also defined as the prioritization technique. In this, the prioritization used in software management and development aims to achieve a common understanding among stakeholders about the importance they attach to the delivery of each requirement, so the acronym that designates: Must have; It should have; It could have, and it will not.

Prioritization involves assigning a priority for each requirement, which usually classifies it as high, medium, or low. This is usually a factor that can generate conflicts, whether of interest or necessity, in a view that each plaintiff sees as unique only their needs. Thus, the need to give transparency to the prioritization of requirements in the search for sensitivity among the plaintiffs is shown.

\subsection{Public Administration}

This subsection has described the study on public administration because the research is about prioritizing requirements in the Attorney General's Office of the State of 
Pernambuco (PGE/PE), government agency, which has its own and distinct characteristics of the private sector in matters of stakeholders, part management, and public relations.

According to Brad (2006), there are many ways to identify key stakeholders. One question raised by the author is: "How much attention does each interested party deserve or demand?" since it is unlikely that everyone involved in various requirements has the same interests, even if they are claimants of the same body.

Since, according to Brad, the organization's stakeholders are identified, there will always be a fight for attention, whom to give it to, to whom to give more, and to whom not to give. Thus, not prioritizing the needs of one party over others is an issue that many organizations face every day.

Based on Post's studies (2002), stakeholders are defined as stakeholders in the requirements, that is, any group or individual affected or that may affect the achievement of an organization's objectives. However, also, according to the author, the definition can be expanded to include groups that have interests in the organization, regardless of the corporation's interest in them.

Also, according to Post, the public designates stakeholders in relationships, for example, employees, shareholders, leaders, etc. These audiences can be segmented by various organizational factors.

The studies here also specify how the public administration usually places its requirements, usually as urgent, and the urgency is usually characterized in two conditions. When a claim is time-sensitive and the second when that claim is important or critical to stakeholders. The Emergency, then, requires a timely response. Urgency alone may not predict the priority of one interested party, especially if the other two attributes are missing. However, this attribute adds a dimension that is particularly important because the urgent public is often of senior management.

Thus, the public administration has an urgent vision for its requirements. For these to be treated with the proper priority and transparency among all organ components is a path to its equalization.

\section{Action Research Method}

The research method used in this study was research-action. According to Carr (2006), action research has its origins associated with early interventionist practices carried out by Kurt Lewin in the 1940s, during socio-technical experiments. The initial stimulus for the emergence and design of the main objectives and aspirations of action research came from a generalized difficulty at that time in translating the results of social research into practical actions.

According to Sjoberg et al. (2007), the action research method was pointed out as "the type of study where the most realistic scenario is found" because it involves applying results of academic studies in a natural context of the industry. 
To explicitly describe the objectives of action research and guide its steps, the following research questions were defined: What difficulties are identified to prioritize the requirements? What are the benefits of adopting a prioritization approach in the context of the public organization?

\subsection{Research Context}

Supported by the Systematic Mapping of Ahmad's Literature, Ahmad et al. (2018) and articles on the implementation of Action Research, as published by Dos Santos and Travassos (2011) and Davison, R. et al., this study aims to demonstrate the results of a research-action carried out at PGE/PE for the implementation of an online Kanban tool. The expectation is that this online tool will provide improvement in the transparency of the activities of the development team and facilitate the prioritization of requirements by senior management.

According to Anderson (2010), Kanban properties are Visualization: allow visual monitoring of requirements; Progress: follow the construction steps; Flow: inform the order of execution of the steps; Policies: be aware of the rules; Improvement: notify changes; Prioritization: to give the science of the order of execution of the implemented requirements; Transparency: allow stakeholders to track all implemented properties online. This research action was based on the implementation of two properties: Prioritization and Transparency.

The Attorney General's Office of the State of Pernambuco (PGE/PE) is the body responsible for the judicial representation of the State of Pernambuco and its municipalities, including in its competence the activities of legal advice to the Executive Branch and promotion of active debt collections, promoting measures of a legal nature aimed at protecting public assets and defending the State, among other activities State of Pernambuco (1990).

The development team is formed by systems coordination, digital automation and innovation, currently with a team of nine people, which is part of the structure of the computer area of the attorney general, subordinated to the General Secretariat. In its 11 years of operation, the coordination of systems has already developed more than 400 applications for the use of PGE/PE, mostly applications for registrations, management control, or process automation. As the volume of requirements has been increasing exponentially, PGE/PE felt the need to adopt a tool to promote the control and management of requirements because daily new requirements arise in the attorney general's office concerning new systems and requests for improvement of existing ones.

Because all incoming requests are urgent, it is difficult for the team to define the priority order assigned to each activity. The main problem encountered today concerns the dissemination or plural display of the requirements, the priorities that each activity has, and the impacts that will be generated with non-compliance with the requirements, respecting the deadlines previously established. 
The tool via the web allows monitoring results in real-time, in the same way for all involved. Another advantage is the ability to track and analyze work with monitoring the runtime of requirements and reports.

The online tool for the Kanban frame method, according to the readings of the referenced jobs, is an application that can increase team productivity and visual project management. It assists in managing tasks and projects with colored notes in a virtual Kanban frame, allowing the use of the computer itself to view, control, and optimize workflow and collaborate with other team members in real-time. A unique feature of an electronic Kanban board is the opportunity to monitor and analyze the work with built-in metrics, which in the traditional format, must be performed daily or weekly. In addition, web-based Kanban frames can be easily customized to map any workflow.

PGE/PE has already had experiences with Kanban practices manually to promote internal control of development team requirements. With this use, some method deficiencies were observed concerning the display and prioritization of the requirements. Some researchers, such as Anderson (2010) and Petersen (2014), have already observed these deficiencies concerning Kanban in Software.

It is believed that conducting a research action to evaluate the use of an online Kanban tool will allow the discovery of new practices already applied by the community, which can help solve or minimize the impacts currently felt by the lack of transparency and prioritization of requirements in PGE /PE.

\subsection{Research Steps}

According to Santos e Travassos (2011), action research consists of five basic steps: Diagnosis, Action Planning, Action Taken, Evaluation, and Lessons Learned. This research work was conducted using the Santos and Travassos guide (2011).

Two research cycles were carried out-action: (1) The first cycle provided vital information to the company of the current situation and presented the perception of employees about the possibility of using Kanban as a tool to manage software requirements with greater assertiveness and transparency; (2) The second cycle put into practice the lessons learned in the first making the necessary adjustments to achieve the objective.

Cycle 1 Diagnosis: Currently, the system coordination team receives requirements from all areas without a hierarchical central point, which hinders the prioritization work carried out by the systems coordinator because as the plaintiffs are chief prosecutors, all their requirements are essential.

Action planning: The preparation of the questionnaire was performed, and the potential respondents were defined. The objective of this study was to record the knowledge that the developers have about the Kanban method and how much they believe that the tool can be applied in the daily activities of PGE/PE generating speed in the visualization and definition of priorities. 
Action taken: Application of the questionnaire to the technical team and consolidation of the results obtained. From the analysis of the results obtained in the questionnaire, it was identified the need to prepare a new questionnaire to be applied to senior management. The object of this was to legitimize the need to apply the transparency technique to prioritize the requirements. Four questions were applied. The questionnaire was answered by the following attorneys: General Attorney, Deputy General Attorney, Chief Attorney, and the General Secretary.

The first questionnaire was answered by nine people from the systems team. About the participants, it is essential to highlight that $40 \%$ have a complete degree, and $60 \%$ are currently attending the undergraduate course.

Evaluation: In the first questionnaire, when respondents were asked about knowing the Kanban method, $60 \%$ reported knowing fully, while $40 \%$ reported knowing partially. On the possibility of Kanban applicability in the current company, $80 \%$ agreed that Kanban could be applied, while $20 \%$ partially agreed. When asked about believing that using Kanban would improve their work, $60 \%$ partially agreed, while $40 \%$ agreed.

In the second questionnaire, when respondents were asked about knowing the requirements being performed by the development team, $33.3 \%$ answered that they know, and $66 \%$ know partially. Regarding the importance of specialized stakeholders having visibility of tasks to prioritize the requirements of the development team, $83.3 \%$ agree that it is essential, while $16.7 \%$ do not. Regarding believing that the use of an online tool that demonstrates the activities of the development team can contribute to the prioritization and transparency of tasks, 83.3\% answered yes, and $16.7 \%$ answered partially. Regarding knowing the Kanban method, $100 \%$ of stakeholders answered no.

Lessons learned: The need to apply the questionnaire to senior management had not been initially identified. This need was only felt from the information collected in the first questionnaire. Thus, the importance of analyzing the data correctly and allowing changes in planning, whenever possible, is emphasized.

Cycle 2 - Diagnosis: During the execution of the application of requirements management with prioritization through the online tool, some difficulties were identified, such as Adaptation of the manual process to the electronic process; Lack of culture to update the data in the tool informing the evolution of the states of the development process; The non-access by the senior management of the tool for online monitoring of the evolution of requirements and their prioritizations; Problems with the browser, we have identified instability in that browser.

Action planning: The processes for implementing the online tool were defined: Registration of information in the tool; Start date of use of the tool; Training to the systems team and senior management.

From the use of the Kanban method in an online tool, it is believed that the information and requirements that will reach the PGE/PE systems sector will be filtered with greater assertiveness and will be visible to all stakeholders. 
The action was taken: To solve the problems mentioned in the diagnosis, the following procedures were mapped: To solve the lack of updating of the data in the tool, the coordinator began to monitor daily and charge.

Tool update: a procedure was created for the automatic display of the tool on the Intranet to allow the monitoring of the high management of the requirements and their prioritization, thus facilitating the access of stakeholders to the information.

Evaluation: In the execution of the second cycle, it was possible to verify that the learning curve of the use of the tool was better because all involved already had the necessary knowledge of the tool.

As a positive aspect, we can highlight that Kanban can be used as an embedded system. The stakeholders who made use of this version very much liked the possibility of this rapid monitoring.

Lessons learned: The applicability of an online tool made it possible to verify the need for conditioning of use by both the technical team and the claimants. Consequently, a new activity was generated for coordination, which is the daily verification of the updating of properties by the technical team and senior management.

\section{Results}

After implementing the Kanban tool, used in this research action to contribute to the visibility and prioritization of the requirements in PGE/PE, excellent results could be perceived. The fact that the systems team already knows the tool helped reduce the learning curve and facilitated the achievement of the objectives. In the first cycle, it is already possible to perceive results obtained using the tool.

Although there are prosecutors who disagree with the visualization of the requirements and their priorities, because of the organization's culture and they have, we expect that this proposed awareness will bring good results and speed to the internal processes of the systems team.

Another aspect that we report as an initial result is the update of the required state and the immediate interaction by the plaintiffs, as electronic state change warnings are sent after the completion of each phase. This functionality aroused in the team greater motivation and interest in knowing better the tool adopted because other functionalities can be identified to add more value to the use of the tool.

\subsection{Discussion}

It is essential to understand that a tool to monitor the requirements of software in development can quickly allow the visualization of priorities and progress of requests, inhibiting abrupt interruptions in the development of applications routinely, which causes tremendous impacts and delay in the process of re-conducting development, especially those that were in the most advanced stage. 
Over time, some applications lost their practical use, as they lost the entry window into production due to other requirements that arrived later, but received the sieve of priorities, even when the time for completion of the development of the requirement already in its final phase of development and provides for approval.

The application of Kanban had already shown positive results concerning the understanding by the entire development team of what each is involved in. This allowed for the rapid change of components between requirements when one had to be absent for reasons. However, as everyone is following the requests of the whole team, there is a collective awareness of the work being carried out. This gain was something not expected by the coordination but positive in her analysis.

As for the aspect of awareness of senior management, it can already be verified that the number of interactions with the secretary-general has been decreasing because he can now do online monitoring of requirements and only interfere when he thinks prudent. This allowed the smallest number of face-to-face meetings, which resulted in more coordination present with the team and thus gave the necessary support to it.

\subsection{Threats}

After applying the questionnaire to the high management of the attorney general's office, it can be verified that not all high management prosecutors share the idea of the benefit of transparency of the prioritization of requirements and the visibility of all the requirements. After a meeting with the respondents, it appears that the general secretary who responds as the information technology superintendent of the PGE/PE, since there is no such structure in the body, the same came to agree and encourage the use of this approach, since this function has the objective of monitoring, monitoring, and managing in a macro way the technology requirements of the agency.

Some chief prosecutors of the specialized did not positively visualize the exposure of their requirements to the others specialized, since, according to them, each attorney has its own needs that do not correlate with the needs of others, so they do not see the need for exposure of their requirements and actions to all.

It can be noted that the Attorney General, Deputy Attorney General, Secretary General, and the Chief Attorney agreed to have the requirements transparent and accessible to senior management moreover, that some coordinating attorneys do not visualize this transparency with some positive impact or change generator that makes the work more efficient. However, for the coordination of systems, the implementation of this approach is significant, as it will allow the immediate negotiation with the SecretaryGeneral of the interruption or not of development to meet other requirements that arrived later.

We can say that the main threats mapped were: A survey was conducted at the level of senior management, and in this, it was found that not all prosecutors agreed that sharing the requirements is positive; The lack of intimacy with software development project management technologies by some prosecutors may generate a lack of interest in the use of the tooling; The high volume of requirements and their distribution among a 
team with small composition can generate an imbalance in the prioritization of requirements.

\section{Conclusion}

Based on bibliographic studies that sought the combination of Kanban themes, prioritization, requirements, and public administration, we identified the opportunity to apply this research action in the Attorney General's Office of the State of Pernambuco.

As the construction of software in the attorney's office follows the agile development line, the use of Kanban has suited the organization's needs. Kanban is based on five properties, according to Anderson (2010): visualization, progress; flow; policies; and improvement. As the focus of the research was the search for the solution to the problem of workflow breakage, it was necessary to search for the addition of one more property, that of prioritization.

In the proposed panel, resulting from the evolution of what was already used with what was being implemented, we observed that the properties needed to be complemented. Then we performed the addition of two properties, prioritization, and transparency. To promote prioritization and transparency efficiently, we apply, from the research activities, the Kanban method through an online tool to manage PGE/PE requirements.

With the use of the tool, it was possible to direct the workflow, allowing stakeholders to analyze the process and delivery in real-time in addition to allowing the analysis of the flow in a measured and managed way, since the control can now be carried out through the tool by all involved, quickly allowing the plaintiffs' participation.

The consequence of this action is that there has been a collaboration between the plaintiffs, thus avoiding overlapping requirements and flow breaks since all start to have co-participation in the requirements of the coordination of the agency's system.

Thus, it can be inferred that the most significant gain of the application of this process was the union of development efforts with the knowledge of the plaintiffs' business, which is the high management of the attorney general's office and the interaction between them. Collaboration between the areas, which by nature are distinct and have unique needs, was also allowed, but in the macro view, they seek to solve a single problem, which is to generate economic for the State, to recover the active debt, and to provide legal support to public processes.

Thus, as final considerations, with this work, it was possible to verify that Kanban can be used in software development and management, using an online tool for teams with few resources and many requirements. With Kanban, it was possible to create a sequence of jobs based on prioritizations. The significant gain of the study was to promote a greater interaction of the plaintiffs in the iterations of each phase of the project, avoiding the breakdown of the development flow with inadequate prioritizations. When an activity is prioritized in the wrong way, it can generate negative impacts on the general results of the organ and discomfort among stakeholders. 


\section{References}

Hujainah, F. Bakar, R. B. A. Abdulgabber, M. A. and Zamli, K. Z. (2018) "Software requirements prioritization: A systematic literature review on significance, stakeholders, techniques and challenges," IEEE Access, vol. 6, pp. 71497-71523.

Babar, M. I. Ramzan, M. and Ghayyur, S. A. K. (2011) "Challenges and future trends in software requirements prioritization," in International Conference on Computer Networks and Information Technology, pp. 319-324.

Anderson, D. (2010) Kanban: Successful Evolutionary Change for Your Technology Business. Blue Hole Press. [Online]. Available: https://books.google.com.br/books?id=RJ0VUkfUWZkC

Petersen, K. e. a. (2014) "Early identification of bottlenecks in very large-scale system of systems software development".

Taibi, D. e. a. (2017) "Comparing requirements decomposition within the scrum, scrum with Kanban," International Conference on Agile Software Development, p. 68-83.

Lehtola, L. e. a. (2004) "Requirements prioritization challenges in practice," Product focused software process improvement, pp. 497-508.

Karlsson, J. e. a. (1997) “A cost-value approach for prioritizing requirements," IEEE Software September/October, pp. 67-74.

Berander, P. e. a. (2005) "Requirements prioritization," Engineering and managing software requirements, pp. 69-94.

Beck, K. (2000) Extreme Programming Explained: Embrace Change. Addison-Wesley.

McIntyre, J. (2016) “MoSCoW or kano - how do you prioritize?” HotPMO.

Brad, L. (2006) "Prioritizing stakeholders for public relations," Institute for Public Relations.

Post, J., e. a. (2002) "Managing the extended enterprise: The new stakeholder view," California Management Review, pp. 6-28.

Carr, W. (2006) "Philosophy, methodology and action research," Journal of philosophy of education, vol. 40, no. 4, pp. 421-435.

Sjoberg, D. I. Dyba, T. and Jorgensen, M. (2007) "The future of empirical methods in software engineering research," in 2007 Future of Software Engineering. IEEE Computer Society, pp. 358-378.

Ahmad, M. O. Dennehy, D. Conboy, K. and Oivo, M. (2018) "Kanban in software engineering: A systematic mapping study," Journal of Systems and Software, vol. 137, pp. 96 - 113. [Online]. Available:

http://www.sciencedirect.com/science/article/pii/S0164121217302820

Dos Santos, P. S. M. and Travassos, G. H. (2011) "Action research can swing the balance in experimental software engineering," in Advances in computers. Elsevier, vol. 83, pp. 205-276. 
of the State of Pernambuco, G. (1990), "Complementary Law n.2," https://legis.alepe.pe.gov.br/texto.aspx?id=5127tipo=TEXTOATUALIZADO.

Santos, P. and Travassos, G. (2011) "Action research can swing the balance in experimental software engineering," Advances in computers, vol. 83, pp. 205-276.

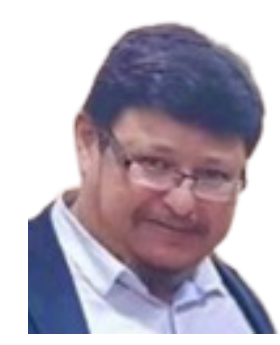

Shot bio: Álvaro Farias Pinheiro is an Analyst in Information and Communication Technology Management (AGTIC) of the State Agency of Information Technology and Communication of the State of Pernambuco (ATI/PE), Systems Coordinator, Digital Automation, and Innovation of the Attorney General's Office of the State of Pernambuco (PGE/PE) and Director of Institutional Relations of the Association of Servers of the State of Pernambuco (ASSERTI). He is studying his $\mathrm{PhD}$ in Computational Intelligence and Software Engineering from the School of Engineering of The Polytechnic of Pernambuco of the University of Pernambuco (UPE). He holds a master's degree in Software Engineering from the Center for Advanced Studies and Systems of Recife (CESAR). He has a specialization in Software Engineering Development Methodologies by the Brazilian Union of Technology (UNIBRATEC). He holds a bachelor's degree in Information Systems with an Emphasis on Software Engineering from the Integrated College of Recife (FIR). He received a bachelor's degree in Computer Science from the Catholic University of Pernambuco (UNICAP). 